\title{
A COMPLETENESS THEOREM FOR A NONLINEAR PROBLEM
}

\author{
by K. J. BROWN
}

(Received 6th July 1973)

\section{Introduction}

As is well known, the linear Sturm-Liouville eigenvalue problem on a bounded real interval $[a, b]$ possesses a family of eigenfunctions which is a complete orthonormal system for the real Hilbert space $L_{2}[a, b]$, i.e. there exists a sequence of eigenfunctions $\left\{u_{n}\right\}$ such that $\left(u_{i}, u_{j}\right)=\delta_{i j}$ (Kronecker delta) for $i, j \in \mathbf{N}$ (the set of positive integers) and, if $u \in L_{2}[a, b], u=\sum_{j=1}^{\infty} c_{j} u_{j}$ where $c_{j}=\left(u, u_{j}\right)$. Pimbley $(4, \mathrm{p} .113)$, raises the question as to whether similar completeness results hold for nonlinear problems. In this note we show that certain nonlinear Sturm-Liouville eigenvalue problems possess eigenfunctions which form a basis for $L_{2}[a, b]$, i.e. there exists a sequence of eigenfunctions $\left\{v_{n}\right\}$ for the nonlinear problem such that every $u \in L_{2}[a, b]$ can be expressed in the form $u=\sum_{j=1}^{\infty} c_{j} v_{j}$ by means of a unique sequence $\left\{c_{n}\right\}$ of real numbers.

Before stating our main result, we must first recall some properties of linear Sturm-Liouville problems. If $\mathbf{R}$ denotes the set of real numbers, let $p:[a, b] \rightarrow \mathbf{R}$ be continuously differentiable with $p(x)>0$ for $x \in[a, b]$ and let $q:[a, b] \rightarrow \mathbf{R}$ be continuous. Consider the equations

$$
\begin{gathered}
-\left(p u^{\prime}\right)^{\prime}(x)+q(x) u(x)=\lambda u(x), \\
a_{1} u(a)+a_{2} u^{\prime}(a)=0=b_{1} u(b)+b_{2} u^{\prime}(b) ; a_{1}^{2}+a_{2}^{2} \neq 0, b_{1}^{2}+b_{2}^{2} \neq 0 .
\end{gathered}
$$

Let $L: D(L) \rightarrow L_{2}[a, b]$ be such that $L u=-\left(p u^{\prime}\right)^{\prime}+q u$ where $u \in D(L)$ if and only if $u$ satisfies (2), $u$ is absolutely continuous on $[a, b]$ and

$$
-\left(p u^{\prime}\right)^{\prime}+q u \in L_{2}[a, b] \text {. }
$$

Then $L$ is a self-adjoint operator on $L_{2}[a, b]$. Since $L$ is closed, $D(L)$ is a Banach space with respect to the norm $\|u\|=\|u\|+\|L u\|$ where \|\| denotes the norm in $L_{2}[a, b]$.

With the above notation equations (1) and (2) may be expressed as

$$
L u=\lambda u
$$

We study nonlinear perturbations of equation (3). We shall prove the following:

$$
\text { E.M.S.-19/2-M }
$$


Theorem 1. Let $N_{i}: D(L) \rightarrow L_{2}[a, b]$ be continuously Fréchet differentiable with $N_{i}(0)=0$ and $N_{i}^{\prime}(0)=0$ for $i=1,2$. Then there exists a sequence of eigenfunctions $\left\{v_{n}\right\}$ for the problem

$$
L u+N_{1} u=\lambda\left(u+N_{2} u\right)
$$

such that $\left\{v_{n}\right\}$ is a basis for $L_{2}[a, b]$.

\section{Proof of Theorem 1}

Before proving Theorem 1 we state as propositions the two main facts on which the proof depends.

Proposition 1. Let $\left\{u_{n}\right\}$ be a complete orthonormal system for a Hilbert space $H$. If $\left\{v_{n}\right\}$ is a sequence of vectors in $H$ such that $\sum_{j=1}^{\infty}\left\|u_{j}-v_{j}\right\|^{2}<1$, then $\left\{v_{n}\right\}$ is a basis for $H$.

Proof. See Kato (3), V 2.20 and the subsequent remarks.

Secondly we require a result from bifurcation theory due to Crandall and Rabinowitz (1). Let $A: D(A) \rightarrow L_{2}[a, b]$ be a densely defined closed linear operator on $L_{2}[a, b]$. Then $X=D(A)$ is a Banach space with respect to the norm $\|u\|_{X}=\|u\|+\|A u\|$. Proposition 2 is a special case of Theorem 2.4 in (1).

Proposition 2. Let $N_{i}: X \rightarrow L_{2}[a, b]$ be continuously differentiable and $N_{i}(0)=0$ and $N_{i}^{\prime}(0)=0$ for $i=1,2$. Regarding $A-\lambda_{0} I$ as a map from $X$ to $L_{2}[a, b]$, suppose that $N\left(A-\lambda_{0} I\right)$, the null space of $A-\lambda_{0} I$, is one dimensional and $R\left(A-\lambda_{0} I\right)$ has codimension one i.e. there exist $u_{0} \in X$ and $y_{0} \in L_{2}[a, b]$ such that $N\left(A-\lambda_{0} I\right)=\operatorname{span}\left\{u_{0}\right\}$ and $R\left(A-\lambda_{0} I\right)=\left\{y \in L_{2}[a, b]:\left(y_{0}, y\right)=0\right\}$. If $\left(y_{0}, u_{0}\right) \neq 0$, and $Z$ is any complement of $\operatorname{span}\left\{u_{0}\right\}$ in $X$, then there exists $a$ neighbourhood $U$ of $\left(\lambda_{0}, 0\right)$ in $\mathbf{R} \times X$, a real interval $(-a, a)$ and continuous functions $m:(-a, a) \rightarrow \mathbf{R}$ and $l:(-a, a) \rightarrow Z$ such that $m(0)=\lambda_{0}, l(0)=0$ and the set of all solutions of $A u+N_{1} u=\lambda\left(u+N_{2} u\right)$ contained in $U$ is

$$
\left\{\left(m(s), s u_{0}+s l(s)\right) \in \mathbf{R} \times X:|s|<a\right\} \cup\{(t, 0):(t, 0) \in U\} .
$$

We can now give the

Proof of Theorem 1. By the linear Sturm-Liouville theory there exists an increasing sequence of eigenvalues $\lambda_{1}<\lambda_{2}<\lambda_{3}<\ldots$ and a corresponding sequence of eigenfunctions $u_{1}, u_{2}, u_{3}, \ldots$ for equation (3). Let $k \in \mathbf{N}$. Then

$$
N\left(L-\lambda_{k} I\right)=\operatorname{span}\left\{u_{k}\right\}, R\left(L-\lambda_{k} I\right)=\left\{u \in L_{2}[a, b]:\left(u_{k}, u\right)=0\right\}
$$

and so we can apply Proposition 2 with $\lambda_{0}=\lambda_{k}$ since $\left(u_{k}, u_{k}\right) \neq 0$. In the notation of Proposition 2 but replacing $l, m$ and \|\|$_{X}$ by $l_{k}, m_{k}$ and $\|\mid\|$ respectively, there exists $\delta_{k}>0$ such that $\left\|l_{k}(s)\right\|<1 / 2^{k+1}$ if $|s|<\delta_{k}$. Choose and fix $\alpha_{k}$ such that $0<\left|\alpha_{k}\right|<\delta_{k}$ and let $v_{k}=u_{k}+l_{k}\left(\alpha_{k}\right)$. Since 


$$
\sum_{j=1}^{\infty}\left\|u_{j}-v_{j}\right\|^{2}=\sum_{j=1}^{\infty}\left\|l_{j}\left(\alpha_{j}\right)\right\|^{2} \leqq \sum_{j=1}^{\infty}\left\|f l_{j}\left(\alpha_{j}\right)\right\|^{2} \leqq \sum_{j=1}^{\infty} 1 / 2^{j+1}<1 \text {, }
$$

by Proposition $1,\left\{v_{n}\right\}$ is a basis for $L_{2}[a, b]$ and so $\left\{\alpha_{n} v_{n}\right\}$ is also a basis for $L_{2}[a, b]$. Since, by Proposition 2, $\alpha_{k} v_{k}$ is an eigenfunction for (4) corresponding to the eigenvalue $m_{k}\left(x_{k}\right)$, we have proved that $L_{2}[a, b]$ has a basis consisting of eigenfunctions of (4).

\section{Applications}

(a) The hypothesis that $N$ maps $D(L)$ into $L_{2}[a, b]$ is more easily satisfied than the hypothesis that $N$ maps $L_{2}[a, b]$ into itself. For example, if $N x$ is a polynomial in $x$ the former hypothesis is satisfied but the latter is not. Hence, if $c_{i}:[a, b] \rightarrow \mathbf{R}$ is continuous and $k_{i} \in \mathbf{N}, k_{i}>1$ for $i=1,2, \ldots, n$, then Theorem 1, with $N_{1}=0$ and $N_{2}=0$ respectively, shows that there exist bases $\left\{v_{n}\right\}$ and $\left\{w_{n}\right\}$ for $L_{2}[a, b]$ such that $\left\{v_{n}\right\}$ and $\left\{w_{n}\right\}$ consist of eigenfunctions of

and

$$
-\left(p u^{\prime}\right)^{\prime}(x)+q(x) u(x)=\lambda\left(u(x)+\sum_{i=1}^{n} c_{i}(x)[u(x)]^{k_{i}}\right)
$$

$$
-\left(p u^{\prime}\right)^{\prime}(x)+q(x) u(x)+\sum_{i=1}^{n} c_{i}(x)[u(x)]^{k_{i}}=\lambda u(x)
$$

respectively, satisfying boundary condition (2).

(b) It is clear from the proof that Theorem 1 will hold for appropriate nonlinear perturbations of any unbounded self-adjoint operator $L$ on a Hilbert space $H$ if $L$ possesses a complete orthonormal system of eigenfunctions corresponding to simple eigenvalues. In particular the theorem is applicable in the case of perturbations of a linear Sturm-Liouville problem with discrete spectrum on the interval $[0, \infty)$.

Consider the differential expression $-u^{\prime \prime}+q u$ on $[0, \infty)$ where $q:[0, \infty) \rightarrow \mathbf{R}$ is continuous and $\lim _{x \rightarrow \infty} q(x)=\infty$. Let $L: D(L) \rightarrow L_{2}[0, \infty]$ be such that $L u=-u^{\prime \prime}+q u$ where $u \in D(L)$ if and only if $u \in L_{2}[0, \infty], u^{\prime}$ is absolutely continuous on $[0, T]$ for all $T>0,-u^{\prime \prime}+q u \in L_{2}[0, \infty]$ and $u(0)=0$. Then

(i) since $q$ is bounded below, $-u^{\prime \prime}+q u$ is limit point, i.e. $L$ is selfadjoint (Everitt (2));

(ii) since $\lim q(x)=\infty, L$ has discrete spectrum (Titchmarsh (6));

(iii) if $u \in \stackrel{x \rightarrow \infty}{D(L)}$, then $u^{\prime} \in L_{2}[0, \infty]$. (Everitt (2), Section 5.)

If $u \in D(L)$, then $u^{\prime} \in L_{2}[0, \infty]$ and $u(0)=0$ and so it can be shown that (see, for example, Stuart (5), Proposition 2.3) $u \in L_{p}[0, \infty]$ for $p>2$. Hence, if $c_{i}:[0, \infty) \rightarrow \mathbf{R}$ is bounded, $k_{i} \in \mathbf{N}$ and $k_{i}>1$ for $i=1,2, \ldots, n$,

$$
N: u \rightarrow \sum_{i=1}^{n} c_{i} u^{k_{i}}
$$


satisfies the hypotheses of Theorem 1 and so there exists a basis for $L_{2}[0, \infty]$ consisting of eigenfunctions of

$$
-u^{\prime \prime}(x)+q(x) u(x)=\lambda\left(u(x)+\sum_{i=1}^{n} c_{i}(x)[u(x)]^{k_{i}}\right) ; u(0)=0
$$

and a basis for $L_{2}[0, \infty]$ consisting of eigenfunctions of

$$
-u^{\prime \prime}(x)+q(x) u(x)+\sum_{i=1}^{n} c_{i}(x)[u(x)]^{k_{i}}=\lambda u(x) ; u(0)=0 .
$$

\section{Acknowledgement}

This paper was written while I was on leave of absence at the University of Sussex. I should like to thank the University of Sussex for its hospitality and the Science Research Council for the financial support which made this leave of absence possible. I should also like to thank Dr M. Thompson for drawing my attention to Proposition 1.

\section{REFERENCES}

(1) M. G. Crandall and P. H. Rabinowitz, Bifurcation from simple eigenvalues, J. Functional Analysis 8 (1971), 321-340.

(2) W. N. Everitr, On the limit point classification of second order differential operators, J. London Math. Soc. 41 (1966), 531-34.

(3) T. Kato, Perturbation theory for linear operators (Springer Verlag, 1966).

(4) G. H. Pimbley, Eigenfunction branches of nonlinear operators and their bifurcations (Springer Verlag, 1969).

(5) C. A. Stuart, Some birfurcation theory for $k$-set contractions, Proc. London Math. Soc. 27 (1973), 531-550. 1946).

(6) E. C. Trtchmarsh, Eigenfunction expansions, Part 1 (Oxford University Press,

HERIOT-WATT UNIVERSITY

EDINBURGH 